\title{
Sentimentos e experiências na vida das mulheres com câncer de mama
}

\author{
FEELINGS AND EXPERIENCES IN WOMEN WITH BREAST CANCER'S LIFE
}

SENTIMIENTOS Y EXPERIENCIAS EN LA VIDA DE LAS MUJERES CON CANCER DE MAMA

Carolina Pasquote Vieira', Maria Helena Baena de Moraes Lopes², Antonieta Keiko Kakuda Shimo³

\section{RESUMO}

Identificar os sentimentos e as experiências relacionados ao câncer de mama torna-se importante para que as pessoas de seu convívio social compreendam essa etapa da vida da mulher. Uma revisão da literatura foi realizada buscando identificar quais são os pensamentos e os sentimentos mais comuns experimentados pelas mulheres depois do diagnóstico de câncer de mama. Foi realizada por meio de busca bibliográfica no LILACS e MEDLINE, e as palavras-chave utilizadas foram: breast, câncer, feeling, female, representações, sentimentos. Conteúdos do câncer foram abordados, como o significado da doença e suas implicações para a vida emocional e social da mulher. Concluiu-se que as experiências relacionadas ao câncer de mama têm um âmbito muito individual, tendo representações diferenciadas para cada mulher.

\section{DESCRITORES}

Neoplasias mamárias. Emoções.

Relações interpessoais.

\section{ABSTRACT}

Identifying feelings and experiences related to breast cancer becomes important to enable those who are part of a woman's social circle to grasp this stage of her life. For this study, a literature review was carried out in order to identify what are the most common thoughts and feelings women experience after a breast cancer diagnosis. The review was conducted through bibliographical research in LILACS and MEDLINE databases using the key words breast, cancer, feeling, female, and representation. Contents related to cancer were searched, such as the meaning of the disease and its implication to the woman's emotional and social life. The conclusion was that experiences related to breast cancer have an individual scope, and that their representations are different for each woman.

\section{RESUMEN}

Identificar los sentimientos y experiencias relacionados al cáncer de mama son importantes para que las personas en su alrededor social comprendan esa etapa de vida de la mujer. Una revisión de la literatura ha sido realizada buscando identificar cuales pensamientos y sentimientos son más comunes en las mujeres después de saber del diagnóstico de cáncer de mama. Fue hecha una búsqueda bibliográfica en el LILACS y MEDLINE, y las palabras clave usadas han sido: brease, cáncer, feeling, female, representaciones, sentimientos. Contendidos del cáncer han sido abordados, como el significado de la dolencia y sus implicaciones para la vida emocional y social de la mujer. Se concluye que las experiencias relacionadas con el cáncer de mama tienen un ámbito individual, con sus representaciones diferenciadas para cada mujer.
1 Psicóloga. Mestranda em Enfermagem pela UNICAMP.

ninapvieira@bol.com.br

2 Professora Doutora do Programa de PósGraduação do Departamento de Enfermagem da Faculdade de Ciências Médicas da UNICAMP.

3 Enfermeira. Professora Doutora do Programa de PósGraduação do Departamento de Enfermagem da Faculdade de Ciências Médicas da UNICAMP. akkshimo@ fcm.unicamp.br

\section{KEY WORDS}

Breast neoplasms.

Emotions.

Interpersonal relations.

\section{DESCRIPTORES}

Neoplasias de la mama.

Emociones.

Relaciones interpersonales. 


\section{INTRODUÇÃO}

Compreender as representações que as mulheres acometidas pelo câncer de mama têm acerca de sua doença é algo considerado extremamente relevante. Esta revisão bibliográfica é parte constituinte de um projeto de mestrado, de maior amplitude, que está sendo realizado com o objetivo de entender as crenças que uma equipe de enfermagem tem sobre o cuidado com mulheres com câncer. Portanto, para uma melhor apreensão destas crenças, e dos comportamentos das enfermeiras, inicialmente se faz necessário compreender o perfil das mulheres acometidas pelo câncer de mama, o que sentem acerca de seu diagnóstico e os aspectos psicossociais envolvidos nesse processo. Como o câncer feminino pode se manifestar em diferentes órgãos, foi necessário optar por apenas uma de suas categorias para que este trabalho pudesse ser realizado com êxito.

As representações são aqui compreendidas como um conjunto de idéias, saberes e sentimentos incorporados pelos indivíduos que são provenientes de uma estrutura social mais ampla $^{(1)}$. Também se considera importante avaliar quais são as alterações sociais na vida destas mulheres, bem como os possíveis conflitos enfrentados na sua vida cotidiana como um todo.

Acredita-se que o tema seja de grande relevância para a área da saúde de forma geral, para todos os profissionais que trabalham com pacientes oncológicos, uma vez que pouco se discute sobre esta temática no cotidiano do trabalho. É importante conhecer quem efetivamente é o sujeito do cuidado, para que a assistência possa ser oferecida de forma eficaz e satisfatória.

As pacientes com câncer de mama vivenciam experiências de dor física e psicológica durante diferentes estágios da doença. Ainda assim, não é possível afirmar que todas sintam as mesmas dores, já que este é um conceito subjetivo. As experiências emocionais vividas particularmente influenciam em todo este processo da doença: desde a aceitação, até o tratamento, bem como na qualidade e intensidade da dor. A dor crônica é um processo que envolve diferentes aspectos do ser humano, como cultural, psicológico e social. Além do que, esta é uma experiência desagradável e que traz incômodos de diferentes ordens ${ }^{(2)}$.

O câncer de mama envolve basicamente a passagem por três etapas que se sobrepõem: o recebimento do diagnóstico de estar com câncer (sentido como algo de natureza negativa), a realização de um tratamento longo e agressivo, e a aceitação de um corpo marcado por uma nova imagem com a necessidade de aceitação e convivência com a mesma ${ }^{(3)}$.

O câncer traz mudanças efetivas na vida da pessoa, porque o diagnóstico altera a condição anteriormente estabelecida de atividade para colocá-la num lugar de passividade em relação à vida. Por esses e outros motivos, é muito importante que um tempo seja fornecido ao paciente e à família para que possam lidar com o diagnóstico(4).

Portanto, o câncer de mama precisa ser pensado em toda essa amplitude. A mulher acometida por essa doença não tem apenas o seu corpo modificado, mas também a sua imagem corporal e diferentes aspectos da sua vida social e afetiva.

\section{OBJETIVO}

Identificar quais são os pensamentos e os sentimentos mais comuns vivenciados por mulheres depois do diagnóstico de câncer de mama, por meio de uma revisão de periódicos indexados dos últimos 5 anos.

\section{MÉTODO}

Trata-se de uma revisão da literatura acerca dos aspectos emocionais e sociais que envolvem desde a descoberta do câncer de mama até a possível vivência da mastectomia. As buscas bibliográficas foram realizadas privilegiando os últimos cinco anos (1999-2003), porém três artigos científicos encontrados fora deste período foram utilizados por serem de grande relevância para a elaboração do texto. As referências encontram-se indexadas na LILACS e MEDLINE, tendo sido incluídas apenas as apresentadas na língua portuguesa, inglesa e espanhola. As palavras-chave utilizadas foram: "breast", "câncer", "feeling", "female", câncer, mama, representações, sentimentos. Quatro textos utilizados não estão indexados nas bases citadas acima, mas encontram-se disponíveis no acervo da biblioteca da UNICAMP, tendo sido encontrados através de buscas na página eletrônica da universidade.

Duzentos e cinquienta e seis artigos foram encontrados, adotando-se, portanto, alguns critérios de exclusão. Os textos que se referiam a algum lugar específico foram desconsiderados, devido à relevância dada aos aspectos culturais envolvidos (ex: mulheres de Berlim), bem como aqueles que se referiam a uma etnia específica (ex: mulheres negras). Aqueles em que o enfoque era sobre a família também foram desconsiderados, já que o objetivo é compreender a mulher acometida pela doença. Cento e trinta artigos da literatura médica foram encontrados, com explicações sobre a doença, algumas alternativas de tratamentos e medidas preventivas, como exames mamários e práticas de terapias alternativas. Estes, bem como os textos de prevenção e ações de controle foram excluídos. Artigos que continham relatos apenas de uma determinada faixa etária (ex: mulheres idosas) também foram desconsiderados, já que as análises dos mesmos faziam referência a aspectos específicos das etapas de vida, com características muito peculiares. 
Alguns artigos inicialmente selecionados não foram incluídos devido à dificuldade de acesso, por não serem localizados pelo COMUT e também não estarem disponíveis em sua íntegra na BIREME. Somente foram analisados os textos disponíveis nas bibliotecas da UNICAMP e aqueles que se encontram disponíveis na Internet. Foram encontrados disponíveis por estes recursos (on-line e biblioteca da universidade) 29 textos, e destes, 16 foram selecionados para a elaboração do presente trabalho. Dois livros foram utilizados devido à sua importância para a elaboração de alguns conceitos específicos, e ambos encontram-se disponíveis na biblioteca da universidade.

Durante a leitura dos artigos, pôde-se identificar alguns aspectos sobre o câncer de mama sendo tratados sob diferentes enfoques. Os textos foram analisados e as categorias seguintes puderam ser elaboradas, a fim de facilitar a compreensão e apreensão dos conteúdos relevantes.

\section{RESULTADOSE DISCUSSÃO}

\section{Estar doente}

Quando se pensa em doença, independente do órgão acometido e dos efeitos causados no organismo pela mesma, há um conjunto de sentimentos que se encontram diretamente associados. O momento em que uma pessoa recebe um diagnóstico geralmente é decisivo em sua vida, porque a partir de então, tem a possibilidade de reformular aspectos importantes de sua vida ${ }^{(5)}$.

Algumas consequiências relacionadas ao diagnóstico do câncer estão associadas aos aspectos sociais, outras ao psiquismo, como as idéias recorrentes de morte, o medo da mutilação, da perda de algumas pessoas de seu convívio. Considera-se que a manifestação de uma doença é a demonstração de que outros sintomas estão presentes na vida da pessoa e não estão sendo passíveis de resolução. Existem fatores que estão associados à manifestação do câncer, como por exemplo, vivências de isolamento, histórias de relacionamentos difíceis, vínculos que foram fortemente estabelecidos com pessoas na vida adulta e que são quebrados, incapacidade ou dificuldade de demonstrar sentimentos. No caso do câncer de mama, temse que as mulheres que já apresentam uma personalidade depressiva têm mais chances de desenvolver a doença ${ }^{(6)}$.

O ser humano não está acostumado a tomar contato com sua finitude, e uma das formas de lidar com esta é o fato de usar a fuga. Quando passamos por uma situação de adoecimento o sentimento mais comum é a angústia, porque a morte faz-se presente. Adoecer é uma ameaça à auto-imagem e à existência de todas as pessoas. O sentimento de ansiedade vai aumentando conforme as consultas médicas vão ocorrendo, e alguns pacientes tendem a utilizar os mecanismos de defesa inconscientes para lidar com a situação. Inicialmente negam a doença e desenvolvem a crença de que a cirurgia será o fator principal de resolução das questões de conflito. No entanto, cada pessoa necessita de um tempo particular e subjetivo para lidar com as conseqüências desse diagnóstico e estabelecer formas de lidar com isso ${ }^{(7)}$.

\section{O câncer de mama: questões da identidade feminina}

Quando se descobre com câncer de mama, a mulher adquire uma nova identidade ${ }^{(8)}$. Isso porque o seio é o órgão do corpo feminino que está associado ao prazer e à vida. É ele que fornece leite após a gestação, sendo fonte de alimento para seu bebê. Normalmente, é um símbolo de valor, sendo insubstituível nos caso em que ocorrem perdas ${ }^{(9)}$. O seio é também um símbolo de fertilidade e saúde e durante todas as etapas da vida da mulher, desde a infância, puberdade, até a vida adulta, é o órgão que está mais relacionado à questão da feminilidade ${ }^{(10)}$.

O seio é tido como o objeto central de desejo e satisfação, e adquirir uma doença localizada neste objeto destrói todas as possibilidades de simbolização da mulher enquanto ser feminino ${ }^{(11)}$. Quando é ameaçada da perda deste órgão, a mulher sente que sua identidade feminina está sendo questionada, bem como a sua capacidade para amamentação e sua sensualidade ${ }^{(12)}$.

Mas, as modificações corporais são sentidas mesmo que a paciente não passe pelo processo de mastectomia, porque identifica que há algo estranho dentro de seu corpo. Com a possível retirada de uma ou ambas as mamas, as questões se agravam, porque há uma confrontação real no lugar do que antes era somente simbólico. Lidar com alterações corporais, principalmente porque são relacionadas ao seio, é algo muito difícil para a mulher, independente da faixa etária em que se encontra ${ }^{(9)}$.

Apesar disso, as mulheres que passam pelo processo de remoção do seio criam uma concepção de que não mais precisam se preocupar com a doença, como se o problema estivesse unicamente no órgão, e tendo ele extirpado, todas as questões relacionadas ao câncer estariam sob controle. São nestes pontos que a família e os profissionais de saúde desempenham grande importância no movimento de aceitação da paciente, e no entendimento de que apesar da possibilidade que algumas mudanças ocorram para se adaptar à sua nova condição, a qualidade de vida pode ser mantida ${ }^{(8)}$.

\section{Sentimentos mais freqüentes}

O fato de o próprio indivíduo ser responsável pelo desenvolvimento do cânceré algo diretamente associado às suas vivências, e à qualidade das experiências de vida que estabelece ${ }^{(10)}$.

Durante toda a vivência do câncer, os sentimentos mudam muito. Há um aprendizado muito grande no sentido de buscar uma organização de sua vida, para saber o que vai ser feito para não perder o controle da situação ${ }^{(8)}$.

A associação do câncer com sentimentos negativos como depressão, raiva, tristeza, dor, desespero é comum, bem como a 
sensação de que as pessoas não entendem o sofrimento pelo qual se está passando, o que aumenta a vivência de solidão ${ }^{(7)}$.

O câncer de mama desestrutura a mulher no sentido de trazer para a sua convivência a incerteza da vida, a possibilidade de recorrência da doença e a incerteza do sucesso do tratamento. Uma mulher com câncer busca, durante as diferentes etapas da sua doença, atribuir algum tipo de significado àquilo que está acontecendo com ela. Isso porque os sentimentos que são trazidos juntamente com o diagnóstico são de natureza negativa, como a culpa ${ }^{(13)}$.

As mulheres que passam por uma mastectomia têm uma experiência diferente daquelas que não se submetem a este processo. O sentimento mais comum após a cirurgia é a ambivalência, pois a mulher deposita na cirurgia a possibilidade da cura e espera que, após a realização da mesma, não precise mais se preocupar. Ao mesmo tempo, existe o medo de enfrentar um corpo que já não é mais o mesmo, a sensação de que a doença vai voltar e as necessidades de se preparar para as novas etapas, como os curativos que devem ser feitos. A realidade da mutilação traz para a mulher uma quantidade grande de sentimentos, com os quais ela se sente perturbada, e muitos sentimentos psicológicos de ordem negativa podem surgir nesta etapa ${ }^{(14)}$.

Alguns estudos demonstram que, de forma geral, as mulheres que já tinham algum tipo de desordem emocional antes da descoberta da doença estão mais propensas a desenvolvê-las depois do diagnóstico do que aquelas que nunca tiveram nenhuma ocorrência ${ }^{(15)}$.

O câncer, de forma geral, é uma doença vista como destruidora, geralmente sentida pelas pessoas como um castigo, como uma punição, porque envolve sentimentos difíceis de serem administrados, principalmente o estigma social de morte ${ }^{(11)}$. Dificilmente a mulher que passa pela experiência do câncer de mama retoma sua vida normalmente. As seqüelas existem porque ocorre uma mudança de identidade, já que a auto-imagem não é mais a mesma e a forma como entendem, sentem e interpretam o mundo também mudou ${ }^{(10)}$.

\section{Os aspectos psicossociais do câncer de mama}

Muitas construções culturais acerca do diagnóstico da doença ainda fazem com que a mulher sinta que está recebendo uma sentença de morte. Surgem então o medo de ser mutilada, o medo de rejeição, dentre outros. As representações associadas ao câncer são, na sua grande maioria negativas, e associadas a algo cruel, destrutivo. As diferenças sentidas pela mulher não são apenas no nível corporal, mas também no convívio social, abrangendo família, amigos e trabalho ${ }^{(10)}$. Por isso, é importante para a mulher sentir que tem uma rede de apoio social, que não a permite desistir, e que torna mais fácil o enfrentamento da doença. Normalmente a família ocupa esse lugar ${ }^{(8)}$.

Em relação à fase de reabilitação, a presença do parceiro sexual é altamente significativa, no que se refere à criação de um ambiente saudável para que a mulher possa se sentir novamente integrada no contexto familiar. As mudanças que podem ocorrer na rotina precisam ser incorporadas por todos os membros da família, desde o momento do diagnóstico. É também muito importante que o parceiro esteja apto a oferecer afeto, assim a paciente se sentirá acolhida e compreendida pelo mesmo ${ }^{(16)}$.

A sensação de ter adquirido uma vida ruim, de pior qualidade é muito comum nas mulheres que passaram pela cirurgia de retirada da mama, já que acreditam que a sua vida sexual não será mais a mesma e que os aspectos sociais ficaram muito prejudicados. Já as mulheres que conservam as mamas, apesar do diagnóstico, relatam que a sua qualidade de vida continua satisfatória, mas totalmente associada à imagem corporal ${ }^{(17)}$.

Os sentimentos em relação à família revelam que não há uma alteração extrema no relacionamento entre os membros da família após a descoberta da doença, sendo que aqueles que ocorrem são de natureza psicológica. As vivências são tanto positivas - como o aumento de atenção, de cuidado sentido da parte dos outros - quanto negativas - depressão, isolamento, vergonha, sendo estas respostas das próprias mulheres à doença ${ }^{(17)}$.

Apesar destas constatações, existe a possibilidade da dinâmica familiar ser alterada, uma vez que a mulher sente-se ameaçada e o sentimento de medo começa a ser parte integrante de seu cotidiano. O diagnóstico, assim como as diferentes fases do tratamento trazem importantes consequiências na vida das mulheres. Uma delas é a dificuldade de retomar a sua vida pessoal e também social ${ }^{(10)}$.

O preconceito que existe ao redor do câncer de mama é algo que inviabiliza grande parte das elaborações das mulheres acerca do câncer. $\mathrm{O}$ afastamento de algumas pessoas e as situações de constrangimento, que começam a ser freqüentes, normalmente estão relacionados à não aceitação das pessoas $^{(13)}$.

\section{CONSIDERAÇÕES FINAIS}

Constatou-se que há, na literatura nacional e internacional uma quantidade vasta de artigos científicos publicados a respeito do câncer de mama. No entanto, quando se busca a abordagem emocional e os aspectos sociais envolvidos neste processo, os estudos tornam-se menos frequientes e 
mais difíceis de serem encontrados. Vê-se uma grande necessidade do desenvolvimento maior do tema, já que para se obter uma compreensão acerca das mulheres com câncer de mama, estas precisam ser consideradas em sua integralidade. A doença tem que ser compreendida em sua amplitude, levando-se em consideração que todo ser humano é um ser bio-psico-social. É necessário que mais estudos sejam realizados sob outros enfoques da doença, além da abordagem médica. Os aspectos emocionais envolvidos em todo o processo da doença, desde a descoberta, até a decisão e realização dos tratamentos, são muito importantes na compreensão do que está acontecendo fisicamente na mulher.

O tratamento do câncer feminino precisa ser encarado de forma positiva. É preciso que as representações envolvidas no câncer sejam reformuladas, de forma que ao se defrontar com a doença, a mulher consiga compreender que existem tratamentos eficazes para isto, e que pode ter a sua qualidade de vida de forma satisfatória. $\mathrm{O}$ que acontece é que o diagnóstico está diretamente associado a sentimentos de punição, e isto definitivamente não contribui para a situação da mulher, fazendo com que esta só pense nos aspectos negativos diretamente relacionados ao câncer de mama ${ }^{(11)}$.

Um fator que pode também contribuir para a redução de alguns sentimentos que podem vir a ser experienciados pela mulher é a forma como a notícia do câncer é dada pelos profissionais que a atendem, e qual é o tipo de relacionamento estabelecido com ele. É comum que as mulheres se sintam melhores quando os médicos fazem perguntas não somente associadas ao seu estado físico, mas também quando demonstram algum interesse pela pessoa e pelas vivências emocionais frequientes ${ }^{(18)}$.

Dependendo da fase em que se encontra a mulher portadora de câncer, suas experiências são relatadas de formas particulares e diferenciadas. Alguns autores consideram que o tempo existente entre o diagnóstico e a realização da cirurgia é um fator que deve ser considerado nesta avaliação da doença ${ }^{(19)}$.

\section{REFERÊNCIAS}

1. Queiroz MS. Representações sociais: uma perspectiva multidisciplinar em pesquisa qualitativa. In: Barata RB, BriceñoLeón R. Doenças endêmicas: abordagens sociais, culturais e comportamentais. Rio de Janeiro: FIOCRUZ; 2000. p. 27-46.

2. Silva LMH, Zago MMF. O cuidado do paciente com dor crônica na ótica do enfermeiro. Rev Lat Am Enferm. 2001;9(4):44-9.

\section{CONCLUSÕES}

Conclui-se que as experiências relacionadas com o câncer de mama têm um âmbito muito individual, tendo representações diferenciadas para cada mulher que as vivencia. Mas, o mais importante a ser discutido no momento, é que de acordo com as leituras realizadas, existem muitos sentimentos que são de ordem universal, no sentido de serem comuns a diversas mulheres acometidas pelo câncer de mama, independente da idade e do estado civil. É evidente que para cada situação devem ser consideradas as suas particularidades, levando-se em conta uma mulher acometida pela doença e considerando-se o momento em que esta se encontra.

É importante que se entenda que alguns processos decorrentes da doença são naturais e que é preciso ter paciência e compreensão para que a passagem por esta etapa da vida possa ser facilitada. Também é essencial que as pessoas que vivem com as mulheres que têm câncer de mama sejam capazes de ao menos algumas vezes se remeter ao momento em que se encontram, buscando aceitar as limitações e muitas vezes sendo o apoio necessário (esta atitude é denominada abordagem empática). Todas essas pessoas têm uma importância fundamental na vida destas mulheres, porque muitos sentimentos que estão relacionados ao câncer de mama dependem da forma como serão vistas e recebidas pela família, amigos e profissionais da saúde.

O câncer de mama, nos dias atuais, trata-se de uma patologia altamente significativa, já que torna-se cada vez mais comum entre as mulheres. Os textos analisados referem que algumas questões acerca da doença começam a ser pensadas, mas os conteúdos subjacentes à essa fase de vida da mulher são mais amplos do que aqueles que podem ser encontrados na literatura. $\mathrm{O}$ assunto, devido à sua importância, tem que ser discutido muitas vezes para que as pessoas tenham real consciência do que significa para uma mulher estar acometida de algum "mal" exatamente no órgão que a faz sentir mulher. É uma vivência difícil, cheia de medos e angústias. E a forma de lidar com isso ainda tem que ser descoberta, em cada caso, especificamente.

3. Corbellini VL. Câncer de mama: encontro solitário com o temor do desconhecido. Rev Gaúcha Enferm. 2001;22(1):42-68.

4. Nascimento-Schulze CM. A condição do paciente portador de câncer. In: Nascimento-Schulze CM, organizadora. Dimensões da dor no câncer: reflexões sobre o cuidado interdisciplinar e um novo paradigma de saúde. São Paulo: Robe; 1997. p. 19-30. 
5. Rzeznik C, Dall'Agnol CM. (Re)descobrindo a vida apesar do câncer. Rev Gaúcha Enferm. 2000;21(n. esp):84-100.

6. Ferreira NMLA. O câncer e o doente oncológico segundo a visão de enfermeiros. Rev Bras Cancerol. 1996;42(3):161-70.

7. Miceli AVP. Pré-operatório do paciente oncológico: uma visão psicológica. Rev Bras Cancerol. 1998;44(2):131-7.

8. Bergamasco RB, Angelo M. O sofrimento de descobrir-se com câncer de mama: como o diagnóstico é experienciado pela mulher. Rev Bras Cancerol. 2001;47(3):277-82.

9. Silva RM, Rodrigues DP, Gurgel AH, Farias LM. Auto-exame das mamas em mulheres jovens e a relação com o autocuidado. Rev RENE. 2000;1(1):9-13.

10. Duarte TP, Andrade NA. Enfrentando a mastectomia: análise do relato de mulheres mastectomizadas sobre questões ligadas à sexualidade. Estud Psicol. 2003;8(1):155-63.

11. Gomes R, Skaba MMVF, Vieira RJS. Reinventando a vida: proposta para uma abordagem sócio-antropológica do câncer de mama feminina. Cad Saúde Pública. 2002;18(1):197-204.

12. Arantes SL, Mamede MV. A participação das mulheres com câncer de mama na escolha do tratamento: um direito a ser conquistado. Rev Lat Am Enferm. 2000;11(1):49-58.
13. Almeida AM, Mamede MV, Panobianco MS, Clapis MJ. Construindo o significado da recorrência da doença: a experiência de mulheres com câncer de mama. Rev Lat Am Enferma. 2001;9(5):63-9.

14. Camargo TC, Souza IEO. Atenção à mulher mastectomizada: discutindo aspectos ônticos e a dimensão ontológica da atuação da enfermeira no Hospital do Câncer III. Rev Lat Am Enferm. 2003;11(5):614-21.

15. Bleiker EMA, Hahn DEE, Aaronson NK. Psychosocial issues in cancer genetics. Acta Oncol. 2003;42(4):276-86.

16. Biffi RG, Mamede MV. Suporte social na reabilitação da mulher mastectomizada: o papel do parceiro sexual. Rev Esc Enferm USP. 2004;38(3):262-9.

17. Chapadeiro CA, Sales C, Paiva L, Scandiuzzi D, Anjos ACY. Qualidade de vida de mulheres tratadas de câncer de mama: funcionamento social. Rev Bras Cancerol. 2001;47(3):263-72.

18. Detmar SB, Aaronson NK, Wever LDV, Muller M, Schornagel JH. How are you feeling? Who wants to know? Patients' and oncologists' preferences of discussing health related quality of life issues. J Clin Oncol. 2000;18(18):3295-301.

19. Wallberg B, Michelson H, Nystedt M, Bolund C, Degner L, Wilking N. The meaning of breast cancer. Acta Oncol. 2003;42(1):30-5. 